\title{
La bioética en la investigación de funciones ejecutivas en niños con trastorno específico del lenguaje
}

Manuel Matías Ambiado-Lillo

\begin{abstract}
Resumen: En la actualidad, mucho se habla sobre bioética. Desafortunadamente, en lo que respecta a los lineamientos que deben regular y velar por la dignidad y los derechos de los niños, no existe una coherencia con el nivel de desarrollo técnico contemplado en las investigaciones en el área de los trastornos específicos del lenguaje y las funciones ejecutivas. Para esta investigación se realizaron tres búsquedas diferenciadas en bases de datos de Web of Science, Scopus, Scielo, Biblioteca del Congreso Nacional de Chile y fuentes grises con los descriptores "specific language impairment", "SLI", "executive functions", "memory", "attention", "inhibition", "bioética", "principios de bioética", "bioética infantil", "investigación científica" y "derechos del niño", reparando, específicamente, en la presencia o ausencia de lineamientos éticos declarados en su metodología o procedimiento de trabajo para analizar de forma crítica su progresión desde los años ochenta hasta la actualidad.

En las conclusiones se ha evidenciado que los principios bioéticos generales no son demasiado pertinentes si no se acompañan por políticas nacionales e internacionales que velen por la dignidad de los niños. Por este motivo, contar con disposiciones bioéticas específicas y sistematizadas para la población pediátrica, en la que se incluyen los estudios de FE y TEL, es fundamental. Así es posible abolir todos los dilemas bioéticos que puedan surgir de los vacíos que las actuales leyes presentan. Por esta razón, es indispensable un replanteamiento de principios para este rango etario.
\end{abstract}

Palabras clave: bioética; deontología; trastorno del desarrollo del lenguaje; trastorno del lenguaje; trastorno específico del lenguaje

Recibido: 06/08/2020

Aceptado: 06/08/2021

Disponible en línea: 31/12/2021

* $\quad$ Artículo de investigación

a Licenciado en Fonoaudiología. Magíster en Neurociencias. Diplomado en Filosofía de las Ciencias Cognitivas. Facultad de Ciencias de la Salud. Universidad Arturo Prat. Iquique, Chile. Correo electrónico: mambiado@unap.cl orcid: https://orcid.org/0000-0002-4653-3983 
Cómo citar: Ambiado-Lillo MM. La bioética en la investigación de funciones ejecutivas en niños con trastorno específico del lenguaje. Rev. latinoam. bioet [Internet]. 31 de diciembre de 2021 [citado 31 de diciembre de 2021];21(2):93-106. Disponible en: https://revistas.unimilitar.edu.co/index.php/rlbi/ article/view/5096.

\title{
Bioethics in Researching Executive Functions in Children with Specific Language Impairment
}

\begin{abstract}
Much is said about bioethics. Unfortunately, concerning the guidelines that must regulate and ensure the dignity and rights of children, there is no coherence with the technical development level of research on specific language impairment and executive functions. For this study, three differentiated searches were carried out in Web of Science, Scopus, Scielo, Library of the National Congress of Chile, and gray sources using the descriptors "specific language impairment," "sli," "executive functions," "memory," "attention," "inhibition," "bioética," "principios de bioética," "bioética infantil," "investigación científica," and "derechos del niño." We paid particular attention to the presence or absence of ethical guidelines in the methods or working procedures to critically analyze their progression from the eighties to these days. The conclusions show that general bioethical principles are irrelevant if not accompanied by national and international policies that safeguard the dignity of children. Having specific and systematized bioethical provisions for the pediatric population, including fe and tel studies, will solve all the bioethical dilemmas that may arise from the loopholes in current laws. Therefore, rethinking the principles for this age range is crucial.
\end{abstract}

Keywords: Bioethics; deontology; developmental language disorder; language disorder; specific language impairment

\section{A bioética na pesquisa de funções executivas em crianças com transtorno específico da linguagem}

Resumo: Na atualidade, muito se fala sobre bioética. Infelizmente, no que diz respeito aos lineamentos que devem regularizar e zelar pela dignidade e pelos direitos das crianças, não há uma coerência com o nível de desenvolvimento técnico contemplado nas pesquisas na área dos transtornos específicos da linguagem e das funções executivas. Para esta pesquisa, foram realizadas três buscas diferenciadas em bases de dados de Web of Science, Scopus, SciELO, Biblioteca del Congreso Nacional de Chile e fontes da literatura cinzenta com os descritores "specific language impairment", "sli", "executive functions", "memory", "attention", "inhibition", "bioética", "principios de bioética", "bioética infantil", "investigación científica" e "derechos del niño", salientando, em específico, a presença ou a ausência de lineamentos éticos declarados em sua metodologia ou procedimento de trabalho para analisar de forma crítica sua progressão desde os anos 1980 até a atualidade. Nas conclusões, evidencia-se que os princípios bioéticos gerais não são muito pertinentes se não são acompanhados por políticas nacionais e internacionais que zelem pela dignidade das crianças. Por esse motivo, contar com disposições bioéticas específicas e sistematizadas para a população pediátrica, na qual são incluídos os estudos de fe e tel, é fundamental. Assim, é possível abolir todos os dilemas bioéticos que possam surgir das lacunas que as atuais leis apresentam. Por essa razão, é indispensável uma reconsideração de princípios para essa faixa etária.

Palavras-chave: bioética; deontologia; transtorno do desenvolvimento da linguagem; transtorno específico da linguagem 


\section{Introducción}

En la actualidad mucho se habla sobre bioética. Desafortunadamente, en lo que respecta a los lineamientos que deben regular y velar por la dignidad y los derechos de los niños no existe una coherencia con el nivel de desarrollo técnico contemplado en las investigaciones. Específicamente, en la exposición manifiesta de estos $(1,7)$. Por esta razón, se analizan los antecedentes históricos que sustentan la bioética y cómo, a lo largo de las décadas, se han ido asociando a las investigaciones en el área de los trastornos específicos del lenguaje y las funciones ejecutivas en la población infantil.

En ese orden de ideas, se pone de manifiesto el rol esencial de los principios bioéticos que, sin lugar a dudas, no son suficientes por sí mismos para procurar un resguardo de los niños que forman parte de los grupos de estudio. Por lo que se examinan en relación a los derechos fundamentales internacionales de forma general, y a las disposiciones políticas legales chilenas de forma específica. De este modo, se pretende incentivar la revisión y el análisis de una problemática que está lejos de ser protagonista en la actualidad. Además, se busca destacar el rol activo que juegan los profesionales y los centros académicos al ser los llamados a ampliar este ámbito del saber, tan necesario en tiempos de heterogeneidad en concepciones éticas y morales.

Para esta finalidad, el trabajo se realiza en cuatro etapas. El primer apartado pretende contextualizar al lector en cuanto al marco referencial de los lineamientos bioéticos clásicos que comprenden el desarrollo de los principios de bioética internacional y su aplicación práctica. El segundo apartado expone la transición histórica de la implementación u omisión de estos principios en el estudio de los trastornos específicos del lenguaje y funciones ejecutivas en la población infantil. En el tercer apartado se analiza la realidad contextual chilena, considerando las herramientas legales que regulan la investigación en este rango etario. Por último, el cuarto apartado expone comentarios finales a modo de conclusión sobre la problemática planteada.

\section{Metodología}

Para el levantamiento de información se realizaron tres búsquedas diferenciadas. La primera de ellas consideró la revisión de literatura ligada exclusivamente al tema del "trastorno específico del lenguaje (TEL) y funciones ejecutivas". Para esto, se realizó una búsqueda exhaustiva en la base de datos de la American Speech-Language-Hearing Association (ASHA) por ser la revista de especialidad más longeva y con mayor impacto en cuanto a la especialidad. Se usaron como descriptores "specific language impairment", "SLI", "executive functions", "memory", "attention" e "inhibition". De los 443 resultados encontrados entre 1984 y 2020, se excluyeron todos los que no tenían como objeto de estudio la interacción entre TEL y funciones ejecutivas, obteniendo como resultado un total de 27 artículos. Como complemento se realizó una nueva búsqueda en las bases de datos de Web of Science y Scopus, considerando los mismos descriptores en inglés y español, incluyendo 8 nuevos artículos.

La segunda búsqueda fue realizada por conveniencia en la base de datos de Scielo y de algunas casas de estudios, incluyendo como descriptores "bioética", "principios de bioética" y "bioética infantil". Se obtuvieron un total de 12 resultados pertinentes incluidos en el análisis del escrito. Finalmente, la tercera búsqueda se realizó en la base de datos de la biblioteca del Congreso Nacional de Chile con los descriptores "investigación científica" y "derechos del niño". Obteniendo 3 resultados pertinentes que se suman a los resultados previos.

En cuanto al análisis de la información, se realizó una distribución cronológica de los artículos de trastornos específicos del lenguaje y funciones ejecutivas delimitados por décadas. Esto permitió una visualización progresiva del quehacer científico, reparando específicamente en la presencia o ausencia de lineamientos éticos declarados en su metodología o procedimiento de trabajo. En paralelo, se efectuó una reflexión crítica ligada a los principios de bioética implicados en las investigaciones expuestas. Para finalizar, se llevó a cabo un examen de la realidad chilena contemporánea en la misma línea de investigación. 


\section{Desarrollo}

A lo largo de la historia, el estudio de las funciones cognitivas comunicativas en los niños ha sido un foco de interés para los científicos. Existe una amplia gama de estudios que hacen referencia a los diferentes constructos neuropsicológicos y psicolingüísticos que pretenden dar explicaciones a las intrincadas relaciones existentes entre lenguaje y funciones ejecutivas (FE). En un plano más acotado, y ya desde la década de los setenta, se sitúa a los niños con trastorno específico del lenguaje (TEL) como sujetos de estudios centrales de la investigación que da cuenta de la interacción entre FE y el rendimiento lingüístico.

No obstante, como han ido desarrollándose las líneas de estudios teóricas, es pertinente preguntarse si los lineamientos bioéticos se han desarrollado en paralelo. Probablemente, y por las características históricas, las concepciones bioéticas en las investigaciones de carácter lingüístico cognitivo se presentan de una forma bastante desfasada en el ámbito temporal. Dado que el auge bioético estuvo centrado en las investigaciones que incluían procedimientos invasivos con posibilidades de repercusiones evidentes para sus participantes. Contrastando con los riesgos mínimos que implican las valoraciones lingüístico cognitivas, o por lo menos, no evidentes de forma inmediata.

Al considerar el origen de las normas bioéticas, la historia nos lleva de forma retrospectiva hacia la década de los cuarenta. Específicamente, al año 1946 en el que se firma el Código de Nüremberg (8), documento que plasma para la posteridad que "el consentimiento voluntario del sujeto humano es absolutamente esencial" (9). Este hito de tanta importancia tiene su desafortunado surgimiento a partir de los abusos cometidos por los nazis con los prisioneros. A los cuales utilizaban como sujetos de experimentación sin contar con su consentimiento (8).

Un primer análisis de esta situación podría llevarnos a pensar que no volverán a darse circunstancias similares en las que las vidas de las personas se utilicen como objetos carentes de valor, sensibilidad o conciencia. Pero, para nuestro pesar como especie, la historia nos ha demostrado lo contrario. Pues solo unas cuantas décadas más tarde, un par de nuevos sucesos de gran magnitud con repercusiones negativas nos llevaron a establecer nuevos parámetros bioéticos. Uno de los sucesos que se puede mencionar con respecto a esto son los estudios que pretendían determinar la progresión de la sífilis en la población afroamericana. Los cuales, no exponían la información necesaria que los investigadores tenían a su disposición y, en ese sentido, no consideraban la opción de las personas de consentir o no dicho experimento.

Asimismo, y casi en paralelo, se puede hacer referencia al descubrimiento sobre los niños con discapacidad intelectual institucionalizados que fueron inoculados con virus de hepatitis B como sujetos de pruebas, sin seguir los lineamientos bioéticos (10). Con base en estos hechos, el Departamento de Salud, Educación y Bienestar de los Estados Unidos decidió elaborar un informe que estableciera cuáles eran los principios y guías éticas para la protección de los sujetos humanos de investigación. En el año 1979, finalmente, se publica bajo el nombre de Informe de Belmont (11). El informe contempla tres principios: respeto a las personas, beneficencia y justicia (11). Los cuales, hasta la actualidad, se han transformado en ejes esenciales que sustentan las investigaciones con seres humanos.

Teniendo en cuenta estos principios generales, en 1979 Beauchamp y Childress publican su texto llamado Principles of Biomedical Ethics, en el cual agregan un principio denominado no maleficencia. A partir de allí, la bioética contemporánea se rige por los cuatro principios esenciales para el desarrollo de la investigación (12). Para una adecuada comprensión de las implicancias que tiene la bioética en la investigación de niños con TEL, es de suma importancia conocer a qué hace referencia cada uno de estos principios. De este modo, el lector podrá contar con una base para reflexionar de manera crítica sobre las metodologías y consideraciones que han tenido o que no han estimado los investigadores en este campo.

El primer principio denominado "respeto a la persona" hace referencia a la autonomía (13). Entendiéndose esta como la independencia de los seres humanos para ser partícipes o no de 
actividades que consideren pertinentes por diversos motivos; médicos, psicológicos, religiosos, sociales, etc. Desde esta primera conceptualización, se puede analizar que las personas deben ser capaces de razonar de forma independiente $y$, en base a su proceso reflexivo, estimar si ser o no ser parte de un grupo de estudio.

Un segundo pensamiento podría centrarse en el análisis de las personas que, por diversas razones, no se encuentran facultadas para llevar a cabo un proceso analítico que les permita discriminar entre beneficios y consecuencias de participar en un estudio. Por lo tanto, sería necesario establecer inicialmente dos posibilidades: excluirlos de cualquier grupo de estudio o establecer un representante que pueda ayudar en la decisión, velando por la integridad de dicha persona. Independiente de cual sea la situación personal de cada sujeto de estudio, se puede dimensionar la gran importancia que el consentimiento informado tiene para resguardar el "principio del respeto a la persona".

El segundo principio denominado "beneficencia" se basa en asegurar el bienestar de las personas que, según el Observatorio de Bioética y Derecho de la Universidad de Barcelona (11) equivaldría a dos máximas, "no causar ningún daño, y maximizar los beneficios posibles y disminuir los posibles daños" (p3). De este postulado, alineándose a la primera máxima, podríamos establecer que ninguna investigación se podría llevar a cabo si se considerara desde su concepción que los participantes podrían ser afectados por los procedimientos o las consecuencias de estos.

En cuanto a la segunda máxima, es necesario analizar las posibilidades que permite su enunciado. En primera instancia, al hablar sobre maximizar los beneficios posibles, se está implicando que toda investigación que se realice deberá ser beneficiosa para los participantes. Por lo que sus resultados deberán estipularse a priori para que esta información pueda ser considerada en la toma de decisiones. Por otra parte, al hacer referencia a disminuir los posibles daños, se presume por defecto que existe la posibilidad real de que los estudios conlleven a consecuencias negativas para las personas. Lo cual entra en confrontación directa con la primera máxima que pretende asegurar que las personas no sufran daño alguno en sus participaciones.

En ese orden de ideas, lo correcto sería que el principio de beneficencia no incluyera contradicciones con respecto a otros principios. Si ha de considerarse que alguno puede tener falencias, el menos indicado es este, pues es un principio que pretende velar por el bienestar en ausencia de perjuicios. No se trata de suprimir el costo-beneficio de las investigaciones, elemento siempre presente en todo orden de las cosas, sino más bien de encontrar para cada principio una formulación idónea que no se contraponga al sentido de lo que se pretende proteger.

El tercer principio denominado "no-maleficencia" se describe como la acción de no dañar a las personas. A diferencia del principio de beneficencia de carácter preventivo, en este caso se enmarca la visión desde la perspectiva de actuar siempre bajo la premisa de que ningún procedimiento o decisión debe concebirse a la base de perjuicios sobre los participantes. En ese sentido, se entiende como un lineamiento complementario que apunta a la misma finalidad, pero concebido desde una construcción sintáctica opuesta. Es por este motivo que "algunos filósofos consideran a la no-maleficencia y la beneficencia como un mismo principio" (6, p125).

El cuarto y último principio, al que se le denomina "justicia", hace referencia a la distribución equitativa de los beneficios que puedan derivar de las investigaciones en los tratos igualitarios independiente de las condiciones basales de las personas. Por ello, es un principio que pretende resguardar, sobre todo, a quienes son más carentes y vulnerables en la sociedad en que se encuentran inmersos. A tal efecto, la distribución equitativa podría variar en la entrega de mayores beneficios, de diversa índole, a los más desposeídos (10).

Al tener una visión más clara de los principios, se puede proceder de forma gradual al análisis central de la reflexión. Como se ha mencionado, desde los años setenta se comenzó a estudiar la relación del TEL y las FE, pero, como quedó en evidencia, el desarrollo de las normativas bioéticas se llevó a cabo casi en paralelo. Por esta razón, es comprensible que el foco de interés haya estado más ligado a los estudios invasivos con repercusiones 
inmediatas y evidentes en el bienestar de las personas. En consecuencia, las investigaciones centradas en características cognitivas conductuales quedaron al margen en esta etapa inicial. Al realizar una búsqueda específica y detallada en las bases de datos de la American Speech-Language-Hearing Association, (asociación situada como la más prestigiosa a nivel mundial; referencia en fonoaudiología por su amplia trayectoria y por la rigurosidad en sus procesos de filtrado de información para publicar) se corrobora la observación previa.

Posteriormente, en la década de los ochentas los estudios se centraron específicamente en lenguaje y cognición. Pero en ninguno de los artículos publicados se describió la inclusión del consentimiento informado en su metodología de trabajo ni en sus procedimientos. Solo se restringieron a la selección y valoración de los participantes (1, 2). En los noventas, el panorama siguió siendo desalentador en el ámbito de la bioética. Debido a que se registró un aumento en las investigaciones de niños con TEL, pero no un progreso gradual en la explicitación de los lineamientos bioéticos que se consideran necesarios $(3,7)$ Por este motivo, es destacable que Gilliam, Cowan y Day hayan tomado la iniciativa de expresar a los padres de los participantes las condiciones en las que se llevaba a cabo su investigación, e incluirlo en su publicación.

Los resultados de este análisis siguen la misma línea en la primera década del segundo milenio. Se continuó con la investigación sin explicitar los lineamientos bioéticos $(15,25) 12$ normally developing children matched for chronological age (CA. En este momento de la historia resulta más cuestionable la poca claridad con la que se presentan los estudios en este aspecto. Pues con toda la evidencia a disposición y contando con experiencias previas que pudieron servir como modelos, se siguió invisibilizando el rol esencial de la bioética en este tipo de investigaciones.

En la última década, esta práctica se ha hecho más común $(26,34)$ the verbal inhibition task, and the nonverbal preschoolers with and without specific language impairment shifting task. Comparisons of standardized group (SLI, y es cada vez menos frecuente la revisión de artículos que no cuenten con la declaración de los lineamientos bioéticos $(35,37)$. Es más, poco a poco se ha incorporado la figura esencial de los comités de bioética; organismos encargados de velar por el adecuado proceder en el campo de la investigación $(38,41)$ a higher order executive function skill, in children with specific language impairment (SLI.

Cabe aclarar que la crítica en este artículo apunta a la visibilizar los lineamientos de bioética y no se inmiscuye con la ejecución de las investigaciones. Ya que al leerlas en detalle se infiere que los padres o tutores estaban en conocimiento de los procedimientos que se realizarían a los niños y, por lo tanto, los autorizaron. Sin embargo, esta información debería estar tan bien declarada como lo están los resultados y las conclusiones que los investigadores exponen. En la tabla 1 se exponen de forma sintética las investigaciones referenciadas y sus disposiciones bioéticas.

Tabla 1. Resumen de las disposiciones bioéticas de las investigaciones de FE y TEL abordadas en el manuscrito a lo largo de las décadas.

\begin{tabular}{lll}
\hline 1985 & $\begin{array}{l}\text { Kirchner y } \\
\text { Klatzky }\end{array}$ & No se informan. \\
\hline 1987 & $\begin{array}{l}\text { Nelson, Kamhi y } \\
\text { Apel }\end{array}$ & No se informan. \\
\hline 1990 & $\begin{array}{l}\text { Gathercoley } \\
\text { Baddeley }\end{array}$ & No se informan. \\
\hline 1993 & Van der Lely & No se informan. \\
\hline 1993 & $\begin{array}{l}\text { Masterson, } \\
\text { Evans y Aloia }\end{array}$ & No se informan. \\
\hline 1993 & Masterson & No se informan. \\
\hline 1995 & Montgomery & No se informan. \\
\hline 1995 & $\begin{array}{l}\text { Gillam, Cowan } \\
\text { yay }\end{array}$ & $\begin{array}{l}\text { Se informa a los padres de los } \\
\text { participantes el proyecto de } \\
\text { investigación y se les solicita un } \\
\text { permiso de inclusión. }\end{array}$ \\
\hline 2007 & $\begin{array}{l}\text { Leonard, } \\
\text { Weismer, Miller, }\end{array}$ & No se informan. \\
\hline 2000 & Montgomery & No se informan. \\
\hline 2004 & Montgomery & No se informan. \\
\hline & $\begin{array}{l}\text { Archibald y } \\
\text { Gathercole }\end{array}$ & No se informan. \\
\hline 2006
\end{tabular}




\begin{tabular}{|c|c|c|}
\hline 2007 & $\begin{array}{l}\text { Montgomery y } \\
\text { Windsor }\end{array}$ & No se informan. \\
\hline 2008 & $\begin{array}{l}\text { Spaulding, } \\
\text { Plante y Vance }\end{array}$ & No se informan. \\
\hline 2009 & $\begin{array}{l}\text { Nickisch y von } \\
\text { Kries }\end{array}$ & No se informan. \\
\hline 2009 & $\begin{array}{l}\text { Finneran, } \\
\text { Francis y } \\
\text { Leonard }\end{array}$ & No se informan. \\
\hline 2009 & $\begin{array}{l}\text { Montgomery y } \\
\text { Evans }\end{array}$ & No se informan. \\
\hline 2010 & $\begin{array}{l}\text { Mainela-Arnold, } \\
\text { Evans y Coady }\end{array}$ & No se informan. \\
\hline 2012 & $\begin{array}{l}\text { Magimairaj y } \\
\text { Montgomery }\end{array}$ & $\begin{array}{l}\text { Se obtuvo aprobación de la Junta } \\
\text { de Revisión Institucional. }\end{array}$ \\
\hline 2017 & $\begin{array}{l}\text { Kapa, Plante y } \\
\text { Doubleday }\end{array}$ & $\begin{array}{l}\text { Se obtuvo consentimiento } \\
\text { informado escrito de los padres y } \\
\text { asentimientos de los niños. }\end{array}$ \\
\hline 2017 & $\begin{array}{l}\text { Kaushanskaya, } \\
\text { Park, } \\
\text { Gangopadhyay, } \\
\text { Davidson y } \\
\text { Weismer }\end{array}$ & $\begin{array}{l}\text { Se obtuvo aprobación de la Junta } \\
\text { de Revisión de la Universidad de } \\
\text { Wisconsin-Institución Madison, } \\
\text { además de consentimiento } \\
\text { informado escrito de los padres y } \\
\text { asentimientos de los niños. }\end{array}$ \\
\hline 2017 & $\begin{array}{l}\text { Stokes, Klee, } \\
\text { Kornisch y } \\
\text { Furlong }\end{array}$ & $\begin{array}{l}\text { Se obtuvo consentimiento } \\
\text { informado escrito de los padres y } \\
\text { asentimientos de los niños. }\end{array}$ \\
\hline 2017 & $\begin{array}{l}\text { White, } \\
\text { Alexander y } \\
\text { Greenfield }\end{array}$ & $\begin{array}{l}\text { Se obtuvo consentimiento } \\
\text { informado escrito de padres, } \\
\text { directores y maestros de los niños. }\end{array}$ \\
\hline 2007 & $\begin{array}{l}\text { Buiza-Navarrete, } \\
\text { Adrián-Torres } \\
\text { y González- } \\
\text { Sánchez }\end{array}$ & $\begin{array}{l}\text { Se obtuvo participación voluntaria } \\
0 \text { autorizada por padres y } \\
\text { maestros. }\end{array}$ \\
\hline 2017 & $\begin{array}{l}\text { Lepe-Martínez, } \\
\text { Pérez-Salas, } \\
\text { Rojas-Barahona } \\
\text { y Ramos-Galarza }\end{array}$ & $\begin{array}{l}\text { Se obtuvo consentimiento } \\
\text { informado escrito de los padres. }\end{array}$ \\
\hline 2017 & $\begin{array}{l}\text { Acosta, Ramírez } \\
\text { y Hernández }\end{array}$ & No se informan. \\
\hline 2017 & Yang y Gray & No se informan. \\
\hline 2019 & Ladányi y Lukács & $\begin{array}{l}\text { Se obtuvo consentimiento } \\
\text { informado escrito de los padres. } \\
\text { Se actuó de acuerdo a los } \\
\text { principios de la Declaración de } \\
\text { Helsinki y las estipulaciones de la } \\
\text { junta de revisión local. }\end{array}$ \\
\hline 2019 & $\begin{array}{l}\text { Aljahlan y } \\
\text { Spaulding }\end{array}$ & No se informan. \\
\hline
\end{tabular}

\section{Ambiado-Lillo, Se obtuvo consentimiento Navarroy informado escrito de los padres. Ibáñez-Alfonso}

Fuente: elaboración propia.

La importancia de destacar la exposición de los procedimientos bioéticos en investigación radica en el posicionamiento del sujeto de estudio como una persona autónoma. El cual se impone como prioridad ante cualquier nuevo conocimiento que de ella podría derivar. Por lo tanto, se entiende que, actualmente, la investigación y el progreso están a disposición de las personas, y no las personas al servicio de la investigación como ocurría un par de décadas atrás. De esta idea, nuevamente, surge el cuestionamiento de costo-beneficio que, sin lugar a dudas, es un punto neural en el análisis del progreso y, por ende, de la investigación. Pero por efectos prácticos deberá ser analizado en otra instancia.

Por toda la información expuesta anteriormente, muchas son las preguntas que se pueden plantear desde la perspectiva de la investigación con niños. Algunas que pueden resultar orientadoras son: ¿todos los principios de bioética son pertinentes tanto para niños como para adultos?, ¿existen diferenciaciones en la aplicación de estos en los niños?, ¿son suficientes estos principios?, ¿qué ocurre en el caso de los niños institucionalizados?, ¿qué ocurre en el caso de las familias de los niños con menos recursos socioeducativos? Y, posiblemente, estas se vayan resolviendo, por lo menos de forma parcial, en la progresión de la lectura.

El primer principio enunciado, correspondiente al "respeto a la persona", es muy complejo de ejecutar en las investigaciones de los niños preescolares, rango etario en el que se desarrolla una gran cantidad de estudios, si es que no la mayoría de ellos. La dificultad recae en la autonomía. Más específicamente, en la autonomía cognitiva que, como se ha descrito, está en constante desarrollo, pero no alcanza sus progresos más significativos hasta la educación secundaria, y es en la adolescencia en que se observan más evidencias de esta (42). Por este motivo se establece que frente a situaciones en que los niños no pueden valorar las posibles repercusiones de los estudios en que participan, 
los padres o tutores son las personas que deben determinar la inclusión o no de los niños en las investigaciones.

Ahora bien, en estos casos surge una nueva discusión: ¿qué ocurre si un niño no quiere participar?, ¿se podría obligarlo a participar si se cuenta con la firma del consentimiento informado por parte de los padres?, ¿es suficiente solo la consideración legal? Para dar respuesta a estas preguntas y alineándose al espíritu de la bioética, se plantea el concepto de asentimiento. Como menciona Martínez (10), esta nueva herramienta busca que todo los estudios deban contar no solo con el consentimiento informado firmado por los padres o tutores, sino también por los niños. Ellos deberán ratificar explícitamente esta decisión para que se lleve a cabo el estudio. A su vez, esto se alinea con los derechos internacionales del niño. Específicamente, con lo expuesto en el artículo 12 que versa sobre el derecho a ser escuchado (43).

Con esto en mente y como se ha de entender, para contar con los niños es necesaria una explicación lo más clara y detallada posible de las tareas que se llevarán a cabo en la investigación. Por lo tanto, los investigadores deben valerse de un lenguaje acorde al entendimiento de los menores junto con todas las herramientas que puedan colaborar en el cometido; como videos, imágenes, sonidos, etc.

En relación al "principio de beneficencia", específicamente en la investigación en el área de TEL y FE, si bien se presume que se asegura el bienestar de los participantes, el apartado de maximizar los beneficios es dudoso por lo menos en el 50\% de ellos. Es lógico que a continuación la pregunta sea ¿por qué? Para poder comprender la razón, se debe ir a las bases metodológicas de los estudios del área. Al hacer un análisis breve de la información disponible en cualquier revista de especialidad, por lo menos en el $80 \%$ de los casos, los estudios se realizan con una metodología de trabajo caso-control. Por consiguiente, los beneficios de la investigación tributarán directamente a los casos de los niños con TEL. Pero el grupo control que se constituye por niños con desarrollo lingüístico típico ¿qué beneficios obtiene?, ¿se debe apelar al altruismo en pro del desarrollo de nuevos conocimientos en esta área? Lo más probable es que la respuesta a esta última pregunta sea positiva.

El aporte desinteresado de las personas en pro del avance científico permite el crecimiento y evolución de las sociedades en las que se encuentran inmersas. En ese sentido, la visión para potenciar el desarrollo social estaría basada en los aportes individualizados carentes de beneficios propios. Sin embargo, esta característica positivista no es suficiente para sustentar por sí sola el "principio de beneficencia".

De forma astuta se presenta el "principio de justicia" que, como se ha mencionado previamente, trata de repartir de forma equitativa los beneficios que se podrían obtener como resultado de la investigación. Como ocurre en el "principio de beneficencia", los resultados irían en directo beneficio de los niños con TEL. Considerando esa hipótesis, ¿es justo que los niños con desarrollo lingüístico típico invirtieran tiempo de recreación o de interacción familiar cuando no han de obtener algún provecho de la actividad?

Como último principio general, se debe analizar el "principio de no-maleficencia." En lo que respecta a la investigación en el área, no existe ningún cuestionamiento a la hora de generar los planes de acción para llevar a cabo las investigaciones. Por esta razón, y en sintonía con lo propuesto por Baines en 2008 (44), se decanta por el postulado de que solo el "principio de no-maleficencia" sería ejecutado a cabalidad en la investigación con niños.

Teniendo presente los resultados de los análisis, es oportuno preguntarse si son suficientes los principios generales de bioética para la investigación infantil. A la luz de la evidencia, es claro ver que estos no cubren todas las posibilidades que se contempla en este tipo de estudios. Para lograr una mayor rigurosidad en los procesos de investigación, como se ha mencionado previamente, es indispensable el establecimiento de comités de bioética. Afirmación que concuerda con el planteamiento de Molina (45), quien explicita que investigadores y comités deben realizar un trabajo coordinado, aportando de forma recíproca desde sus especialidades. Así, los investigadores se encargarán de diseñar, proponer y ejecutar estudios relevantes para la sociedad, en tanto que los comités 
prestarán servicios orientadores, reguladores y certificadores para su adecuada aplicación.

Profundizando un poco más con respecto a los comités de bioéticas, estos deberían contar con diversas áreas de agrupaciones a cargo de profesionales especializados en ellas. Algunos ejemplos de estas áreas podrían ser rangos etarios, creencias, ideologías y condiciones médicas basales, etc. Lamentablemente, es una concepción que no corresponde con la realidad de la mayoría de las instituciones. Sea por falta de estructura, de equipamientos o de recursos humanos (46). Por lo tanto, es de suma importancia que los investigadores se adjudiquen esa responsabilidad bioética de dignificar a las personas que participen con ellos. En cuanto a esta disposición, los centros académicos están llamados a ser agentes principales en la difusión y formación de profesionales con una mirada más crítica y analítica sobre aspectos bioéticos y morales que se enmarcan en cada investigación que se realiza.

Con un panorama general claro, solo resta informar la realidad contemporánea de la investigación con niños en Chile. En la actualidad, Chile cuenta con la Ley 20.120 ("sobre la investigación científica en el ser humano, su genoma, y prohíbe la clonación humana") que entró en vigencia el 22 de septiembre del año 2006 (47) y el decreto de la norma que entró en vigencia el 14 de enero del año 2013 (48). El organismo encargado de velar por el cumplimento de esta normativa es el Ministerio de Salud (MINSAL). Los artículos más relevantes de la Ley 20.120 para el análisis que nos compete sobre investigación en niños con TEL y su relación con FE son: Artículo $2^{\circ}$, Artículo $11^{\circ}$ y Artículo $14^{\circ}$ los cuales se citan a continuación.

El Artículo $2^{\circ}$ expresa

"La libertad para llevar a cabo actividades de investigación científica biomédica en seres humanos tiene como límite el respeto a los derechos y libertades esenciales que emanan de la naturaleza humana, reconocidos tanto por la Constitución Política de la República como por los tratados internacionales ratificados por Chile y que se encuentren vigentes" $(27, \mathrm{p} 1)$

Por lo tanto, cualquier investigación que vulnere algún derecho del niño será motivo de sanción para los investigadores e instituciones patrocinantes, si es que las hubiera. Esto se debe a que en el año 1990, el gobierno de Chile suscribió la convención sobre los derechos del niño realizada por la Organización de las Naciones Unidas (ONU) en el año 1989 (49).

\section{El Artículo $11^{\circ}$ expone}

"Toda investigación científica realizada con seres humanos deberá contar con el consentimiento previo, expreso, libre, informado y por escrito de la persona o, en su defecto, con el de aquel que deba suplir su voluntad en conformidad con la ley y en el caso de los niños menores de 18 y mayores de 12 se permitirá, en la medida de lo posible, su presencia al acto para conocer su opinión. El consentimiento deberá constar en un acta firmada por la persona que ha de consentir en la investigación, por el investigador responsable o principal, en su caso, y por el director del centro o establecimiento donde ella se llevará a cabo, quien, además, actuará como ministro de fe. El director del centro o establecimiento podrá delegar la facultad de suscripción del acta donde conste el consentimiento informado y su actuación como ministro de fe" (27, p3).

Sobre este artículo hay mucho que se puede analizar. Inicialmente no se hace alusión a los niños menores de 12 años. Por lo que se puede entender que quedan sometidos forzosamente a la decisión de sus padres o tutores. Esto abre el debate inicial sobre si los menores podrían ser sometidos a estudios, aunque ellos no los hayan aceptado. Afortunadamente, y como se explicita en el Artículo $2^{\circ}$, por ley se exige el respeto de los derechos del niño. Por lo que en caso de negativa en la participación esta será la decisión que prime en la ejecución del proceso. Aún así es preocupante que, al día de hoy, casi una década más tarde, no existan lineamientos normados por ley que establezcan la forma de proceder en investigación pediátrica. Cuando la evidencia y los juicios de expertos apuntan a la gran problemática que se presenta en estos estudios (50).

El artículo $14^{\circ}$ manifiesta

"El consentimiento deberá ser nuevamente solicitado cada vez que los términos o condiciones en que se desarrolle la investigación experimenten modificaciones que tengan incidencia en las 
personas que participan en ella, salvo que éstas sean consideradas menores por el Comité que haya aprobado el proyecto de investigación" (27, p4).

Por este motivo, cada vez que el proceso de investigación deba modificarse, independientemente de la razón que obligue al investigador a hacerlo, los participantes deben ser informados de que nuevamente deberán consentir la participación de los niños. De esto se infiere que los investigadores responsables están en la obligación bioética de no conformarse con el consentimiento de los padres o tutores. Además, deberán volver a informar a los niños las nuevas características que se contemplarán en el estudio para que ellos puedan asentir o no su participación.

Como ha quedado en evidencia, la investigación en niños es un área de mucho interés científico. Pese a esto, el progreso en lineamientos bioéticos está bastante desfasado en cuanto a lo que se requiere para un adecuado control de este tipo de actividades. Por ello la imperiosa necesidad de la difusión temática y los compromisos profesionales.

Para finalizar, es conveniente dar respuesta, en la medida de lo posible, a las preguntas planteadas en un inicio. La primera de ellas: ¿ todos los principios de bioética son pertinentes, tanto para niños como para adultos? Tomando como base los análisis previos, en los que se determinó que, en un sentido práctico y considerando las concepciones semánticas de ellos, solo el principio de no-maleficencia sería adecuado en los niños. Es posible que esto se deba a que los principios se originan desde la experiencia negativa de personas adultas autónomas con las que se experimentó sin su consentimiento.

Otra formulación que se puede intentar responder es: ¿existen diferenciaciones en la aplicación de estos en los niños? Desde lo práctico la aplicación de los principios es igual para todas las personas. Sin embargo, la modalidad de consentimiento es variable. Al considerar solo los aspectos legales las posibilidades que podrían originarse son que los adultos no consientan y los niños asientan o que los adultos consientan y los niños no asientan. En el primer caso, se estaría menoscabando la intencionalidad y, por lo tanto, el desarrollo de autonomía del niño. En el segundo, la situación es aún peor. Se podría obligar a participar a los niños contra su voluntad al contar con el consentimiento de los padres. Lo anterior deja en evidencia la diferenciación bioética en el abordaje de niños y de adultos. Para resolver la siguiente pregunta: ${ }^{\text {son }}$ suficientes estos principios?, se toma como base de análisis este caso. Así, queda de manifiesto la necesidad de que los principios bioéticos generales no son suficientes para resguardar y velar por la dignidad de los niños.

Al reflexionar sobre estas preguntas, se puede entender que, si se habla desde la generalidad de los principios bioéticos en los niños, se presentan múltiples dificultades. En los casos especiales como ¿qué ocurre en el caso de los niños institucionalizados? o ¿qué ocurre en el caso de las familias de los niños con menos recursos socioeducativos?, las respuestas son mucho más complejas de resolver, considerando la inexistencia de lineamientos orientadores de parte de los departamentos gubernamentales.

\section{Conclusiones}

Luego de la exposición de todos los antecedentes generales y específicos se puede concluir que, actualmente, en Chile no existen lineamientos para las investigaciones pediátricas que respalden las directrices bioéticas generales. Sin embargo, para el resguardo de los niños es explí cito que deben ser respetados los derechos infantiles fundamentales propuestos por la ONU en 1989 a los que el gobierno de Chile suscribió en el año 1990.

Además, se ha evidenciado que los principios bioéticos generales no son demasiado pertinentes si no se acompañan por políticas nacionales e internacionales que velen por la dignidad de los menores, dada la vaguedad y la extensión en el análisis al que pueden ser sometidos. A causa de esto, se hace indispensable un replanteamiento de principios para este rango etario de forma específica, que permitan el establecimiento de lineamientos y leyes dirigidas al estudio científico.

Otra conclusión que surge, aunque quizás demasiado a priori, es la ventaja que presentan los estudios que tratan de encontrar la asociación entre 
niños con trastorno específico del lenguaje y su rendimiento en tareas de funciones ejecutivas. Debido a que su naturaleza metodológica no es experimental. Pese a esto, no se descarta la posibilidad de que puedan generar algún tipo de estrés posterior a la investigación. Por este motivo, contar con disposiciones bioéticas específicas y sistematizadas para la población pediátrica, en la que se incluyen los estudios de FE y TEL, se hace fundamental. De esta manera, se pueden abolir todos los dilemas bioéticos que puedan surgir de los vacíos que las actuales leyes presentan. De forma concreta, se pueden mencionar las modificaciones de los protocolos de investigación, la discrepancia entre consentimientos adultos y asentimientos infantiles, la dificultad en la búsqueda de respaldos legales y las posibilidades de acomodar las leyes actuales a favor de los investigadores. Al actuar ante todas estas fallas, sin dudas, las investigaciones en la población de menores mejorarían sustancialmente sus fundamentos bioéticos explícitos. Permitiendo además las posibilidades de replicar criterios y ser un aporte a los comités encargados de visar las investigaciones.

Finalmente, se destaca la importancia de la formación en bioética a razón de que es en los profesionales en quienes recae la responsabilidad de gestar sus proyectos de investigación. Tomando consideraciones que muchas veces no están declaradas bajo las normativas a las que ellos se adscriben. Consideraciones que, dado el contexto y la disponibilidad de recursos de formación teórica actual, deberían fundamentarse más allá del sentido común.

\section{Limitaciones y proyecciones.}

Como limitación en el estudio se debe mencionar que, en la actualidad, la terminología referente al TEL se ha comenzado a reemplazar por la referente al Trastorno del Desarrollo del Lenguaje. Por este motivo, las investigaciones contemporáneas que podrían haber aportado a la discusión no han sido consideradas en la búsqueda inicial. La decisión de utilizar el término Trastorno Específico del Lenguaje (TEL) se fundamenta en que la mayor cantidad de reportes científicos se concentra bajo este rótulo. Lo que permite realizar un análisis cronológico de la progresión bioética a lo largo del tiempo.

La principal proyección del presente análisis se establece en la posibilidad de orientar el desarrollo de nuevos lineamientos bioéticos que consideren la realidad pediátrica en las investigaciones lingüístico-cognitivas. Estos deben responder, de forma concreta, a los principios establecidos y no dejarlos al arbitrio de los equipos investigadores. En la misma línea, se espera que los análisis realizados sirvan como orientaciones para las entidades encargadas de formalizar y sistematizar los requerimientos que la investigación reclama.

\section{Referencias}

1. Kirchner DM, Klatzky RL. Verbal rehearsal and memory in language-disordered children. J Speech, Lang Hear Res. [Internet]. 1985 dic; 28(4): 556-65. Disponible en: https://doi.org/10.1044/jshr.2804.556

2. Nelson LK, Kamhi AG, Apel K. Cognitive strengths and weaknesses in language-impaired children. J Speech Hear Disord [Internet]. 1987 febr; 52(1): 36-43. Disponible en: https://doi.org/10.1044/jshd.5201.36

3. Gathercole SE, Baddeley AD. Phonological memory deficits in language disordered children: is there a causal connection? J Mem Lang. [Internet]. 1990; 29(3): 336-60. Disponible en: https://doi.org/10.1016/0749-596X(90)90004-J

4. Van der Lely HKJ, Howard D. Children with specific language impairment: Linguistic impairment or short-term memory deficit? J Speech Hear Res. [Internet]. 1993; 36(6): 1193-1207. Disponible en: https://doi. org/10.1044/jshr.3606.1193

5. Masterson JJ, Evans LH, Aloia M. Verbal analogical reasoning in children with language-learning disabilities. J Speech Hear Res. [Internet]. 1993 febr; 36(1): 7682. Disponible en: https://doi.org/10.1044/jshr.3601.76

6. Montgomery JW. Sentence comprehension in children with specific language impairment: the role of phonological working memory. J Speech, Lang Hear Res. [Internet]. 1995 febr; 38(1): 187-99. Disponible en: https:// doi.org/10.1044/jshr.3801.187

7. Masterson JJ. The performance of children with language-learning disabilities on two types of cognitive tasks. J Speech Hear Res. [Internet]. 1993; 36(5): 1026-36. Disponible en: https://doi.org/10.1044/ jshr.3605.1026 
8. Anguita V. Guías de consentimiento informado documento de trabajo [Internet]. Santiago de Chile: Universidad Alberto Hurtado; 2016; Disponible en: https://portal.ucm.cl/content/uploads/2016/12/Guias_ de_Consentimiento_Informado.pdf

9. Centro Interdisciplinario de Estudios en Bioética. Santiago de Chile: Universidad de Chile; 2020; El código de Nüremberg. [1 pantalla]. Disponible en: https:// www.uchile.cl/portal/investigacion/centro-interdisciplinario-de-estudios-en-bioetica/documentos/76028/ el-codigo-de-nuremberg

10. Martínez-González C. La actualidad de los principios de la bioética en pediatría. An Pediatría Contin. [Internet]. 2013 en; 11(1): 54-7. Disponible en: doi:10.1016/ S1696-2818(13)70119-7

11. Observatorio de Bioética y Derecho. El informe Belmont [Internet]. Barcelona: Universidad de Barcelona; 2020; Recuperado a partir de: http://www.bioeticayderecho.ub.edu/archivos/norm/InformeBelmont.pdf

12. Mir J, Busquets E. Principios de ética biomédica de Tom L. Beauchamp y James F. Childress. Bioètica \& Debat. [Internet]. 2011; 64(17): 1-7. Disponible en: http://www.ucv.ve/fileadmin/user_upload/facultad_ agronomia/Producion_Animal/ProducciOn_Animal/Bioetica.pdf

13. Siruana JC. Los principios de la bioética y el surgimiento de una bioética intercultural. Veritas. [Internet]. 2010; 22(marzo): 121-57. Disponible en: http:// dx.doi.org/10.4067/S0718-92732010000100006

14. Gillam RB, Cowan N, Day LS. Sequential memory in children with and without language impairment. J Speech Hear Res. [Internet]. 1995; 38(2): 393-402. Disponible en: https://doi.org/10.1044/jshr.3802.393

15. Montgomery JW. Relation of working memory to off-line and real-time sentence processing in children with specific language impairment. Appl Psycholinguist. [Internet]. $2000 \mathrm{mzo}$; 121(1): 117-48. Disponible en: https://doi.org/10.1017/S0142716400001065

16. Montgomery JW. Sentence comprehension in children with specific language impairment: effects of input rate and phonological working memory. Int J Lang Commun Disord. [Internet]. 2004; 39(1): 115-33. Disponible en: doi:10.1080/13682820310001616985

17. Mainela-Arnold E, Evans JL, Coady JA. Explaining lexical-semantic deficits in specific language impairment: the role of phonological similarity, phonological working memory, and lexical competition. J Speech, Lang Hear Res. [Internet]. 2010; 53(6): 1742-56. Disponible en: https://doi.org/10.1044/1092-4388(2010/080198)
18. Archibald LMD, Gathercole SE. Visuospatial immediate memory in specific language impairment. J Speech, Lang Hear Res. [Internet]. 2006 abr; 49(2): 265-77. Disponible en: https://doi.org/10.1044/10924388(2006/022)

19. Spaulding TJ, Plante E, Vance R. Sustained selective attention skills of preschool children with specific language impairment: evidence for separate attentional capacities. J Speech, Lang Hear Res. [Internet]. 2008; 51(1): 16-34. Disponible en: https://doi. org/10.1044/1092-4388(2008/002)

20. Nickisch A, von Kries R. Short-term memory (sтM) constraints in children with specific language impairment (SLI): are there differences between receptive and expressive SLI? J Speech, Lang Hear Res. [Internet]. 2009 jun; 52(3): 578-95. Disponible en: https:/doi. org/10.1044/1092-4388(2008/07-0150)

21. Finneran DA, Francis AL, Leonard LB. Sustained attention in children with specific language impairment (sLI). J Speech, Lang Hear Res. [Internet]. 2009 ag; 52(4): 915-29. Disponible en: https://doi.org/10.1044/10924388(2009/07-0053)

22. Montgomery JW. Verbal working memory and sentence comprehension in children with specific language impairment. J Speech, Lang Hear Res. [Internet]. 2000 abr. 43(2): 293-308. Disponible en: https://doi. org/10.1044/jslhr.4302.293

23. Leonard LB, Weismer SE, Miller CA, Francis DJ, Bruce-Tomblin J, Kail RV. Speed of processing, working memory, and language impairment in children. J Speech, Lang Hear Res. [Internet]. 2007; 50(2): 408-28. Disponible en: https://doi.org/10.1044/10924388(2007/029)

24. Montgomery JW, Windsor J. Examining the language performances of children with and without specific language impairment: contributions of phonological short-term memory and speed of processing. J Speech, Lang Hear Res. [Internet]. 2007; 50(3): 778-97. Disponible en: https://doi.org/10.1044/1092-4388(2007/054)

25. Montgomery JW, Evans JL. Complex sentence comprehension and working memory in children with specific language impairment. J Speech, Lang Hear Res. [Internet]. 2009; 52(2): 269-88. Disponible en: https:// doi.org/10.1044/1092-4388(2008/07-0116)

26. Magimairaj BM, Montgomery JW. Children's verbal working memory: role of processing complexity in predicting spoken sentence comprehension. J Speech, Lang Hear Res. [Internet]. 2012; 55(junio): 669-83. Disponible en: https://doi.org/10.1044/10924388(2011/11-0111) 
27. Kapa LL, Plante E, Doubleday K. Applying an integrative framework of executive function to preschoolers with specific language impairment. J Speech, Lang Hear Res. [Internet]. 2017; 60(8): 2170-84. Disponible en: https://doi.org/10.1044/2017_JSLHR-L-16-0027

28. Kaushanskaya M, Park JS, Gangopadhyay I, Davidson MM, Weismer SE. The relationship between executive functions and language abilities in children: a latent variables approach. J Speech, Lang Hear Res. [Internet]. 2017; 60(4): 912-23. Disponible en: https://doi. org/10.1044/2016_JSLHR-L-15-0310

29. Stokes SF, Klee T, Kornisch M, Furlong L. Visuospatial and verbal short-term memory correlates of vocabulary ability in preschool children. J Speech, Lang Hear Res. [Internet]. 2017; 60(8): 2249-58. Disponible en: https://doi.org/10.1044/2017_JSLHR-L-16-0285

30. White LJ, Alexander A, Greenfield DB. The relationship between executive functioning and language: examining vocabulary, syntax, and language learning in preschoolers attending Head Start. J Exp Child Psychol. [Internet]. 2017; 164: 16-31. Disponible en: https://doi.org/10.1016/j.jecp.2017.06.010

31. Buiza-Navarrete JJ, Adrián-Torres JA, González-Sánchez M. Marcadores neurocognitivos en el trastorno específico del lenguaje. Rev Neurol. [Internet]. 2007; 44(6): 326-33. Disponible en: https://doi.org/10.33588/ rn.4406.2006066

32. Lepe-Martínez N, Pérez-Salas C, Rojas-Barahona CA, Ramos-Galarza C. Funciones ejecutivas en niños preescolares con y sin trastorno del lenguaje. Rev Ecuatoriana Neurol. 2017; 26(3): 197-202.

33. Ladányi E, Lukács Á. Word retrieval difficulties and cognitive control in specific language impairment. J Speech, Lang Hear Res. [Internet]. 2019; 62(4): 91831. Disponible en: https://doi.org/10.1044/2018_JSLHR-L-17-0446

34. Ambiado-Lillo MM, Navarro JJ, Ibáñez-Alfonso JA. Funciones ejecutivas en estudiantes con trastorno específico del lenguaje al comienzo de la escolarización básica. Rev Colomb Psicol. [Internet]. 2020; 29(2): 57-72. Disponible en: https://doi.org/10.15446/rcp. v29n 2.79390

35. Acosta-Rodríguez V, Ramírez-Santana GM, Hernández-Expósito S. Funciones ejecutivas y lenguaje en subtipos de niños con trastorno específico del lenguaje. Neurología. [Internet]. 2017 jul. 1; 32(6): 355-62. Disponible en: https://doi.org/10.1016/j.nrl.2015.12.018

36. Yang H-C, Gray S. Executive function in preschoolers with primary language impairment. J Speech, Lang Hear Res. [Internet]. 2017 febr; 60(2): 379-92. Disponible en: https://doi.org/10.1044/2016_JSLHR-L-15-0267
37. Aljahlan Y, Spaulding TJ. The impact of manipulating attentional shifting demands on preschool children with specific language impairment. J Speech, Lang Hear Res. [Internet]. 2019; 62(2): 324-36. Disponible en: https://doi.org/10.1044/2018_JSLHR-L-17-0358

38. Larson C, Gangopadhyay I, Kaushanskaya M, Weismer SE. The relationship between language and planning in children with language impairment. J Speech, Lang Hear Res. [Internet]. 2019; 62(8): 2772-84. Disponible en: https://doi.org/10.1044/2019_JSLHR-L-18-0367

39. Larson C, Kaplan D, Kaushanskaya M, Weismer SE. Language and inhibition: predictive relationships in children with language impairment relative to typically developing peers. J Speech, Lang Hear Res. [Internet]. 2020; 1-13. Disponible en: https:/doi. org/10.1044/2019_JSLHR-19-00210

40. Roebuck H, Sindberg H, Weismer SE. The role of language in nonlinguistic stimuli: comparing inhibition in children with language impairment. J Speech, Lang Hear Res. [Internet]. 2018; 61(5): 1216-25. Disponible en: https://doi.org/10.1044/2018_JSLHR-L-17-0294

41. Rice ML, Taylor CL, Zubrick SR, Hoffman L, Earnest KK. Heritability of specific language impairment and nonspecific language impairment at ages 4 and 6 years across phenotypes of speech, language, and nonverbal cognition. J Speech, Lang Hear Res [Internet]. 2020 mzo. 23; 63(3): 793-813. Disponible en: https://doi. org/10.1044/2019_JSLHR-19-00012

42. De La Caba-Collado MA, López-Atxurra R. Autonomía: las voces de madres y padres. Rev Educ. [Internet]. 2015; 2015(370): 138-62. Disponible en: DoI:10.4438/1988-592X-RE-2015-370-301

43. Plataforma de Organizaciones de Infancia, editor. Convención Internacional sobre los Derechos del Niño [Internet]. 1989 nov. 20; Nueva York, Estados Unidos. Madrid: Plataforma de Infancia España; 2008. Disponible en: http://plataformadeinfancia. org/derechos-de-infancia/la-convencion-de-los-derechos-de-la-infancia/convencion-sobre-los-derechos-del-nino-texto-oficial/

44. Lozano-Vicente A. Bioética infantil: princípios, questões e problemas. Acta Bioeth. [Internet]. 2017; 23(1): 151-60. Disponible en: http://dx.doi.org/10.4067/ S1726-569X2017000100151

45. Molina-Montoya NP. Aspectos éticos en la investigación con niños. Cienc Tecnol para la Salud Vis y Ocul. [Internet]. 2018; 16(1): 75-87. Disponible en: https:// doi.org/10.19052/sv.4348

46. Almeida Neto JB, Franco TB. Análise das publicações sobre os comitês de ética em pesquisa em Scientific Electronic Library Online (Scielo). Rev Latinoam 
Bioética. [Internet]. 2018; 19(36-1): 25-48. Disponible en: https://doi.org/10.18359/rlbi.3641

47. Ley 20120. Sobre la investigación científica en el ser humano, su genoma, y prohíbe la clonación humana. Septiembre 7 de 2006.

48. Decreto 114 [Ministerio de Salud, Subsecretaria de Salud Pública]. Aprueba reglamento de la Ley 20.120 sobre la investigación científica en el ser humano, su genoma y prohíbe la clonación humana. Noviembre 22 de 2010.

49. Decreto 830 [Ministerio de Relaciones Exteriores]. Promulga la convención sobre los derechos del niño. Agosto 14 de 1990.

50. Sáenz-Cortés M, Escobar-López MT, Aristizábal-Tobler CC. Los menores y adolescentes en la investigación clínica. Tensiones y aspectos éticos. Escritos [Internet]. 2016; 24(53): 345-67. Disponible en: http:// dx.doi.org/10.18566/escr.v24n53.a06 